\title{
Una Estrategia Única para la Promoción de Comunidades de Educación a Distancia: la Escritura de un Diario como Base del Discurso
}

\author{
(A unique Strategy for Promoting Distance Learning Communities: \\ Journal Writing as a Basis for Discurse)
}

\author{
AdDIE M. Johnson \\ Penn State University
}

(EE.UU.)

\begin{abstract}
RESUMEN:El presente informe examina la viabilidad de la escritura de un diario como técnica de docencia en la educación a distancia, concebida para proporcionar una comprensión y un dominio más profundos del contenido del plan de estudios en los cursos de educación para postgraduados. En la tarea de crear unas experiencias de aprendizaje óptimas, pueden utilizarse cuatro clases diferentes de escritura de diarios, en diversas clases de entornos de aprendizaje a distancia; no obstante, el modelo que se muestra se emplea en vídeo/audio-conferencias interactivas de doble dirección. Esta estrategia educativa sirve como base para el discurso de clase y para que los estudiantes tomen parte en debates polémicos y en actividades de resolución de problemas. Los aumentos del contenido pueden llevarse a cabo tanto en entornos sincrónicos como asincrónicos.
\end{abstract}

Estrategias Formativas en Educación a Distancia - El Diario Escrito como Base del Discurso Experiencias de aprendizaje a Distancia Creativas

\begin{abstract}
This paper examines the viability of journal writing as a distance education teaching technique designed to provide deeper understanding and mastery of curriculum content in graduate level education courses. In creating optimal learning experiences, four different types of journal writing can be utilized in various types of distance learning environments, however the model demonstrated is used in two way interactive video/audio conferencing. This instructional strategy serves as a basis for class discourse and student engagement in controversial debates and problem-solving activities. Enhancements dealing with content can be performed in both synchronous and asynchronous environments.
\end{abstract}

Distance Education Instructional Strategy - Journal Writing as a Basis for Discourse Creating Optimal Distance Learning Experiences 


\section{INTRODUCCIÓN}

Antes de planificar un curso nuevo o repetido en el marco de educación a distancia, los profesores a distancia normalmente reflexionan acerca de los cursos anteriores y tratan de determinar la forma de utilizar aquellas estrategias que dieron como resultado unas experiencias educativas óptimas, al tiempo que mantenían el interés del alumno. Incluso aquellos enseñantes que llevan varios años en este campo examinan formas nuevas de ganar el interés del alumno y mejorar su asimilación del contenido del curso. Existe una literatura muy numerosa que discute toda una variedad de excelentes propuestas de educación a distancia, en numerosas disciplinas. Sin embargo, el diseño de enfoques pedagógicos en el ámbito del alumnado a distancia resulta más complejo que en el del aula tradicional, desde el momento en que existen numerosos "obstáculos constatados que hay que superar" (Reiding, 1997, J-8). Aunque muchos investigadores critican la adaptación de técnicas de enseñanza de la educación presencial a la enseñanza a distancia (Knapper, 1988), existen algunas estrategias que son transferibles a la hora de permitir a los alumnos interiorizar la información, con independencia de cuál sea su fuente original, como textos, gráficos, electrónica o grabaciones concebidas para Internet. De igual forma, otros investigadores que se enfrentan al desafío de crear comunidades interactivas de educación a distancia frecuentemente integran toda una variedad de estrategias de enseñanza, tanto tradicionales como "inventivas", para apoyar la enseñanza (Owen, 1989; Gunawardena \& Boverie, 1995; Johnson, 1996). Los usos de la escritura de diarios como estrategia de enseñanza, en una variedad de formatos, representan una adaptación viable del aula presencial al entorno de la educación a distancia.

El presente artículo examina la viabilidad de la escritura de diarios como técnica de enseñanza en la educación a distancia, orientada a proporcionar una comprensión más profunda y un dominio del contenido del plan de estudios en los cursos de enseñanza para graduados. Como práctica que ha alcanzado éxito en la educación a distancia, la ventaja más solicitada de esta estrategia educativa es que intensifica los procesos de comunicación entre el alumno y el profesor, y entre los propios alumnos integrantes de la clase. Se pueden utilizar distintos enfoques, que se describirán más adelante en el presente informe, para interesar a los alumnos en el estudio con profundidad del contenido; el empleo de esta técnica permite a los alumnos "organizar y estructurar el contenido", "relacionar y distinguir las pruebas y el argumento", y "relacionar los conceptos con la experiencia cotidiana" (Brundage et al., 1993). La escritura de diarios proporciona una vía para que los alumnos reflexionen acerca del contenido, se aficionen a la investigación, y compartan reacciones y respuestas de múltiples formas con el profesor, compañeros de clase y/o pequeños grupos dentro de la clase asignada. Esta estrategia aporta realidad a un mundo que de otro modo estaría basado en la investigación. Aunque la descripción de la estrategia educativa está pensada para alumnos adultos en clases de postgrado, los lectores pueden contemplar adaptaciones para sus propias asignaturas. 


\section{ENFRENTARSE AL RETO DEL ALUMNO A DISTANCIA ADULTO}

Una de las premisas básicas de la educación a distancia es el reto de proporcionar una experiencia educativa viable a los alumnos adultos. Existe también la tarea añadida de atraer y mantener a los alumnos adultos ofreciendo trabajos de curso en que se utilicen métodos pedagógicos que satisfagan sus necesidades y modelos de estudio, permitiéndoles así dedicarse a experiencias educativas de una forma que no les impida continuar ocupándose de sus obligaciones familiares y laborales. Algunos de estos retos son mantener el interés del alumno, garantizar el dominio de la materia del curso, conseguir la satisfacción del alumno, superar el aislamiento inherente a la educación a distancia y buscar la dedicación en un proceso de enseñanza centrado en el alumno y basado en preguntas. A la hora de planificar los enfoques pedagógicos del trabajo del curso, sería provechoso que los educadores comprendiesen las características de la mayoría de los alumnos adultos.

Se han llevado a cabo numerosas investigaciones para delinear los "perfiles" de los alumnos a distancia que han tenido éxito y quedado satisfechos, pero no se ha concedido demasiada atención a la importante variable de las "características del alumno individual” (Threkeld \& Brzoska, 1994). Moller (1991), en su modelo, Assure, aboga por que los profesores lleven a cabo un análisis del alumno antes de elaborar el curso de educación a distancia. Este análisis proporcionaría información relativa a las características del alumno, de forma que el diseño del curso podría reflejar los tipos de aprendizaje preferidos por el alumno en la selección de estrategias de enseñanza para su puesta en práctica pedagógica. Moller afirma que la planificación para satisfacer las necesidades del alumno basada en aquellas características del mismo que hayan podido detectarse puede servir también de ayuda para alcanzar la satisfacción y el éxito del alumno. Otro estudio que puede utilizarse es el "Inventario de tipos de aprendizaje" de Hansen, Silver y Strong, basado teóricamente en el "Test de personalidad de Myers-Briggs" (Perrin, 1997). Este inventario permite a los profesores determinar el número de alumnos de la clase cuyos tipos de aprendizaje se identifiquen dentro de las cuatro categorías de directivo, interrogativo, creativo y/o interactivo. La comprensión de la composición de la clase facilita la tarea de orientar las estrategias educativas hacia unos tipos más que hacia otros, al tiempo que se incluyen, en alguna medida, los cuatro tipos. El proporcionar una educación basada en el reconocimiento de las necesidades del alumno estimula la motivación del mismo y favorece su desarrollo académico. Estas áreas representan factores importantes a tener en cuenta a la hora de elaborar los planes de estudio y la pedagogía en la educación a distancia.

Dado que los títulos de las escuelas de postgraduados representan unas credenciales para la promoción en el campo educativo, es más probable que los profesores a distancia de esta disciplina se dediquen a un discurso e investigación 
más serio, para obtener la base de conocimiento necesaria que le ayude a elevar la movilidad de su posición y clase socioeconómica (Heinze, 1983). La investigación revela que la mayoría de los alumnos a distancia de las escuelas de postgraduados son normalmente adultos "maduros, independientes y seguros de sí mismos"... [que] tienen vidas complejas y compromisos significativos laborales y/o familiares... no se pueden trasladar físicamente al campus... están deseosos de aprender y... asumen la responsabilidad de su propio aprendizaje" (Perrin, 1997, p. 15). La mayor parte de los alumnos adultos, de una media de unos 28 años, pagan su propia enseñanza y, por ello, resultan más autodisciplinados, se autogobiernan mejor, y es muy probable que tengan éxito en este medio (Dille \& Mezack, 1991).

Cropley \& Kahl (1983), en su estudio de las características de la educación a distancia, advierten que muchos alumnos adultos poseen ciertos rasgos psicológicos, como por ejemplo ser emprendedores, imponerse su propio ritmo, autoevaluarse, buscar metas y ser capaces de autodirigirse en su aprendizaje" (p. 36). Sus observaciones convalidan otros descubrimientos relacionados con los rasgos de los alumnos a distancia, como por ejemplo una preferencia por las formas de aprendizaje que son autodirigidas y una tendencia al aprendizaje basado en los problemas frente a la pedagogía orientada al plan de estudios (Humphrey, 1974). Estos rasgos ofrecen un retrato de los tipos de alumnos para los que deben diseñar estrategias de enseñanza los profesores, con el fin de facilitar unas condiciones de aprendizaje óptimas.

El objetivo de crear y mantener un entorno de educación a distancia óptimo constituye una tarea imprescindible de planificación y aprendizaje. La mayor parte de la planificación del profesor debe llevarse a cabo mucho antes de los cursos en línea que estén programados, especialmente en aquellas escuelas/universidades que precisen la ayuda de operadores. La preparación para el inicio del curso implica decisiones relativas al establecimiento de sitios web con el contenido esencial, documentos de diseño de página personal, referencias específicas para búsquedas en Internet, creación de gráficos, etc., que son todas tareas que llevan tiempo. La planificación anticipada de la lista de actividades durante una sesión de clase exige la previsión de la distribución del tiempo para cubrir el programa y los posibles retrasos por problemas del equipo. Para aquellos que inicien planes existen abundantes guías con sugerencias de programación en diversas sedes de Internet; por ejemplo, la página web de la Universidad de San Diego redactada por Jodi Reed y Merry Woodruff (1995) proporciona un plan matriz de lecciones y una "lista de control de teleaprendizaje" para ayudar a los educadores a distancia a seguir de cerca la finalización de los detalles esenciales para la preparación de los cursos académicos iniciales. El 'Instructional Development Process' de la Engineering Outreach Website de la Universidad de Idaho ofrece un buen proceso gradual y reflexivo (1995). 
A la hora de resumir esta perspectiva de la planificación para satisfacer las necesidades del alumno adulto, previendo de antemano la miríada de tareas a llevar a cabo antes de la puesta en funcionamiento del curso, el examinar y seleccionar las mejores alternativas para crear unas experiencias de aprendizaje óptimas constituye la principal preocupación cuando se trabaja en el medio de la educación a distancia. No solamente existe la preocupación de explorar las oportunidades de que se dispone mediante el empleo de la tecnología, sino que existe la necesidad de dirigir la enseñanza a un nivel más personal y ciertamente tomando en consideración tanto los dominios cognitivos como afectivos esenciales para que tenga éxito la enseñanza de adultos. Una estrategia básica para intentar servir a los alumnos adultos de forma eficaz en la educación a distancia es poner en práctica técnicas que interesen a los alumnos de tal forma que contemplen el aprendizaje como una experiencia beneficiosa, agradable y que merece la pena. Por ello, el introducir actividades de escritura de diarios y otros tipos de enfoques pedagógicos no tradicionales para graduar experiencias de educación a distancia representa instituir prácticas educativas sensatas en la superación de los retos del entorno de la educación a distancia.

\section{UN MARCO VIABLE PARA PONER EN PRÁCTICA UNA NUEVA TÉCNICA DE APRENDIZAJE}

La descripción que sigue presenta un marco de educación a distancia en el que las estrategias de escritura de diarios se utilizan en un campus universitario. Como parte de una gran universidad, los campus satélites ofrecen a menudo cursos a distancia para postgraduados utilizando sistemas interactivos de vídeo/audio-conferencia a través de 'Picture-tel'. El uso de esta tecnología estimula la respuesta espontánea y apoya la colegialidad y la colaboración entre los alumnos y el profesor. Las últimas características de los equipos digitales de videoconferencia reducidos permiten una completa funcionalidad, con posibilidad de compartir la aplicación con facilidad; los alumnos situados en diferentes sitios pueden trabajar juntos en informes, proyectos y presentaciones en tiempo real. Los curso de la clase completa utilizan fundamentalmente los formatos de interacción sincrónica descritos como "cursos de enseñanza en tiempo real, vivos y similares a la conversación... " (Threkeld \& Brzoska, 1994, p. 46). Las sesiones asincrónicas, consistentes en conversaciones a través del correo electrónico, trabajos en Internet, acceso a la página personal y salas de charla en grupo, proporcionan cobertura individual a las tareas de clase y permiten compartir con el grupo las reacciones y respuestas a los temas del programa, favorecidos por estrategias de escritura de diarios. En los campus pequeños, que operan con una universidad mayor, la utilización del vídeo interactivo en las clases resulta rentable, ya que se compensa con el gran número de alumnos a los que sirve (Ludlow, B., 1994). No obstante, los campus que carecen de tal apoyo pueden encontrarse con que dicha utilización constituye una empresa costosa. 
En los cursos de educación a distancia de la escuela para postgraduados se programan dos sesiones de clase a la semana, con una duración de dos o tres horas cada una, durante siete semanas por período. El personal de ordenadores ayuda a iniciar las sesiones en ambos lugares, después de lo cual el profesor ('home site') y algunos alumnos seleccionados controlan la tecnología en el 'distance site' para comunicarse en persona con los miembros de la clase. Sin embargo, el aspecto más importante de este escenario afecta al reparto de tareas en la clase y cobertura del temario. Este es un entorno que favorece la asignación de proyectos, más que los exámenes finales, para demostrar el conocimiento por parte del alumno tanto del contenido como de la aplicación de la materia objeto de estudio.

Los libros de texto, la pizarra blanca, el correo electrónico y la tecnología de ordenador se utilizan como herramientas de enseñanza en este ámbito de la educación a distancia. Sin embargo, resulta significativa en los procesos de enseñanza y aprendizaje la forma en que se emplean estas herramientas para desarrollar y mantener líneas abiertas de comunicación entre los alumnos y facilitar los diálogos alumno/profesor.

\section{LAS RAZONES DE LA UTILIZACIÓN DE LA ESCRITURA DE DIARIOS EN LOS CURSOS DE EDUCACIÓN A DISTANCIA}

Parecen existir numerosas razones para que la escritura de diarios se implante con éxito en los cursos de educación a distancia. El intercambio de ideas a través del comentario del diario utilizado como base para el discurso de la clase alivia la distancia psico-social prevalente en el trabajo realizado de forma asincrónica (Rezabek, 1997). El uso sincrónico facilita la cohesión de los miembros de la clase por medio de un discurso activo a través de videoconferencia, disminuyendo así la distancia temporal y espacial. La dedicación del alumno a temas polémicos y a actividades de resolución de problemas basadas en el contenido del curso sirve como elemento motivador y humano en las discusiones de clase y aumenta el interés del alumno. Todos estos factores contribuyen a establecer una comunidad de alumnos a distancia, en lugar de un grupo de clase de individuos aislados.

Por ello, el mantener unos buenos procesos de comunicación entre los alumnos de educación a distancia y el profesor es una de las tareas más esenciales a la hora de elaborar los cursos de educación a distancia. En un estudio de una universidad estatal regional del medio oeste, Von Holzen (1995) examinó la capacidad del correo electrónico para apoyar el uso de los diarios de los alumnos, a fin de conseguir un cambio en las actitudes y conocimientos de los alumnos relacionados con los ordenadores. Como resultado de su investigación, llegó a la conclusión de que el uso de un modo asincrónico en esta técnica de enseñanza ayudaba a aumentar la comunicación entre los alumnos y el profesor. Además, los 
alumnos que utilizaron este medio señalaron su preferencia por las comunicaciones a través del correo electrónico frente a los diarios escritos a mano.

El poner en práctica cualquiera de las categorías básicas de reflexiones en la escritura de diarios utilizando un modo asincrónico consistente en compartir el comentario de un diario, establece una base para el discurso del alumno en un formato sincrónico. El discurso basado en el intercambio previo de puntos de vista sobre temas compartidos de forma asincrónica da lugar a unas sesiones de clase de videoconferencia interactiva emocionantes y vivas. Resulta todavía mucho más motivador solicitar de los alumnos que, después de leer el texto de literatura, adopten posturas basadas en una visión particular de un tema y elaboren argumentos desde un punto de vista preestablecido. El aprendizaje basado en los problemas es otra técnica que mantiene al alumno interesado y dedicado al trabajo durante las sesiones de videoconferencia. Esta estrategia, por ello, se presta bien para utilizar ambas modalidades a la hora de diseñar diversas combinaciones de interactividad de alumnos a lo largo de un curso de educación a distancia. También sirve como fundamento para establecer una comunidad de alumnos, dado que los mismos comparten informes importantes basados en temas que evolucionan a través de la discusión de la materia del curso.

Además de estas otras características, la escritura de diarios como estrategia educativa estimula el pensamiento crítico, aumenta la capacidad intelectiva, está centrada en el alumno y le ayuda en su facultad cognitiva. El pedir a los alumnos que analicen el contenido y compartan sus análisis con otros integrantes de la clase permite a los citados alumnos desarrollar unas buenas técnicas de pensamiento crítico y alcanzar una base de conocimiento más amplia (Johnson, 1995). Para aumentar el pensamiento crítico, los alumnos necesitan establecer relaciones entre los conceptos teóricos, abstractos, y las ideas cotidianas concretas (Garside, 1994). Por este motivo, el hacer que los alumnos se dediquen a técnicas de aprendizaje activas y no pasivas permite a los profesores ayudar a la construcción del conocimiento, más que a la transmisión de información.

En términos de desarrollo intelectual, a medida que los alumnos examinan sus reacciones a la literatura del curso a la vista de las perspectivas de los demás, comienzan a integrar y sintetizar información que enriquece su valoración inicial de los temas. Estos procesos sirven para aumentar el pensamiento crítico que, a su vez, genera subsiguientes temas de exploración a través del discurso de la clase; de esta forma, el comentario de los diarios se convierte en el "trampolín" para el planteamiento de problemas y resolución de los mismos. La utilización de problemas ayuda a los alumnos a aprender técnicas de pensamiento explícitas como "analizar un problema conceptualmente, formulando y evaluando diversos modos de buscar soluciones" y a examinar la eficacia de las soluciones (Lowman, 1995, p. 236). La combinación de estos procesos de aprendizaje parece contribuir a un grado más elevado de percepción en el alumno, al exigirle que interiorice la 
información para aplicarla. Además, el proporcionar un entorno para que los alumnos analicen, reflexionen y sinteticen el contenido del curso en vista de su propia base de conocimiento práctico permite un desplazamiento del aprendizaje dirigido por el profesor, para centrarlo en el alumno. La selección de los temas, problemas y preocupaciones por parte del alumno se convierte en el centro tanto del comentario del diario como del discurso compañero/clase (Johnson, 1995). Esta forma de aprendizaje centrada en el alumno ofrece la oportunidad de que los alumnos a distancia desarrollen técnicas tanto en el campo cognoscitivo como en el afectivo.

\section{LA ESCRITURA DE DIARIOS COMO ESTRATEGIA DE ENSEÑANZA EN LA EDUCACIÓN A DISTANCIA}

Previa al inicio de cualquier estrategia de enseñanza es la identificación de qué y cómo quieren los profesores que los alumnos adquieran determinados tipos de conocimiento. Teniendo en cuenta que la investigación citada presenta a estos alumnos ante todo con la característica de ser autónomos por naturaleza y con una manifiesta preferencia por las actividades de resolución de problemas, el determinar un enfoque pedagógico que apoye estos rasgos ayuda a guiar a los alumnos hacia la obtención del conocimiento deseado. Por este motivo, el utilizar la escritura de diarios como técnica de enseñanza/aprendizaje se reconoce como estrategia útil para cubrir las necesidades del alumno a distancia.

Basándose en la información cuya interiorización se desea, los profesores determinan el contenido de las entradas del diario, que pueden consistir en cualquier combinación de las siguientes: (1) una crítica o análisis de la perspectiva de un autor acerca de un tema ofrecido en un texto, gráfico, sitio Internet, etc., (2) una descripción de la importancia de este tema para la experiencia práctica u observada de la propia vida real, o una experiencia hipotética, (3) una solución ofrecida al problema planteado por los autores en el texto y/o (4) el planteamiento de cuestiones basadas en el análisis de las obras del autor. El aspecto más importante de la tarea de escritura de diarios es el establecimiento de una premisa para el discurso en clase, ya sea con un compañero, con un grupo pequeño o con toda la clase.

La utilización de los diarios como medio de reflejar y sintetizar los conceptos fundamentales de la literatura del curso está reconocida como enfoque válido para el conocimiento del alumno (Seshachari, 1994). El compartir los puntos de vista del diario a través de críticas, relacionando las experiencias prácticas con el contenido del curso y desarrollando una investigación para la resolución de problemas basada en el análisis, aumenta la conexión del conocimiento previo con el conocimiento nuevo, facilita el crecimiento intelectual a través del pensamiento crítico y estimula un enfoque del discurso de clase centrado en el alumno. 


\section{CATEGORÍAS DE TÉCNICAS DE ESCRITURA DE DIARIOS}

El empleo de la escritura de diarios se lleva a cabo mediante numerosas formas de diálogo como alumno con profesor, alumno con alumno (diarios de compañeros), intercambio interactivo en pequeños grupos y anotaciones diarias de los alumnos. La selección de la forma de diálogo depende del resultado deseado y se representa en las siguientes categorías.

En la primera categoría, de alumno con profesor, el compartir el diario a través del correo electrónico aumenta la comunicación entre el alumno y el profesor en cuanto a cobertura del contenido, con una realimentación consecuente e inmediata. Aún siendo representativa del pensamiento purista sobre la puesta en práctica de la escritura de diarios para la consecución de objetivos académicos, constituye una tarea que lleva mucho tiempo al profesor, especialmente si la técnica es representativa de un solo tipo de interacción de clase a distancia.

Una categoría preferible es la que involucra a estudiante con estudiante compañero de diario. En esta segunda categoría, los miembros de la clase seleccionan a un compañero basándose en el conocimiento de los intereses del mismo, determinados por un discurso inicial en clase durante sesiones de videoconferencia. Se solicita a los compañeros de diario que reflexionen acerca de partes determinadas del contenido textual del curso y que compartan sus puntos de vista a través del correo electrónico; sus reflexiones son también compartidas por los miembros de la clase durante las sesiones de videoconferencia y sirven de base para la discusión en clase de temas basados en los puntos de vista de los autores. Las opiniones compartidas son finalmente presentadas al profesor para que las revise y les dé respuesta cuando se aproxime el final del período; la longitud de las páginas de respuesta del diario está establecida, a fin de mantener reflexiones iguales y centradas. No obstante, el establecimiento de un foro en línea "hace que los alumnos controlen qué escribir, cuándo... y cómo responder" (Owen, 1983, p. 2). Esta forma de aprendizaje representa un enfoque válido para interiorizar información mediante el examen de temas utilizando el análisis, la reflexión, las opiniones compartidas, la argumentación, el planteamiento de preguntas, el ofrecimiento de soluciones, etc. (Garside, 1994).

Una tercera categoría, consistente en compartir el diario con un pequeño grupo interactivo, puede llevarse a cabo estableciendo unos tiempos determinados para que los grupos de alumnos se dediquen a compartir sus puntos de vista durante una "sesión de sala de charla sobre el diario". Esta modalidad prepara el escenario para el intercambio de opiniones y posturas argumentales, porque se puede pedir a los alumnos que adopten posturas específicas respecto de un tema y que argumenten desde esa perspectiva. Los resultados de las discusiones pueden debatirse después durante sesiones de clase normales de videoconferencia. Estas sesiones más pequeñas preparan el terreno para el debate de todo el grupo, 
orientado a apoyar el pensamiento crítico y creativo, la retención del contenido de la materia y la construcción de nuevos conocimientos. Concebido como foro en el que sirve de intermediario el ordenador, esto formato proporciona un "contexto significativo, basado en el lenguaje, para ofrecer ideas en formato escrito" (Owen, 1983, p. 2).

La última categoría de anotaciones diarias del alumno representa la estrategia menos interactiva. Sin embargo, puede ser útil si se están empleando otras muchas estrategias educativas que ocupen la mayor parte de la sesión de clase de videoconferencia. Se pide a los alumnos que se dediquen a poner en práctica cualquiera de los formatos (o categorías) de escritura de diarios antes mencionados, registrando así sus propios puntos de vista dentro de un marco determinado en una anotación semanal que se remitirá al profesor al final del período. Las anotaciones diarias pueden también utilizarse como base para la discusión en clase con un intercambio limitado durante las sesiones de clase propiamente dicha, puesto que no todos los alumnos van a poder discutir sus puntos de vista. Sin embargo, otro aspecto de toda escritura de diarios no presentado hasta el momento es la petición de que los alumnos reflexionen algo más después de la discusión en clase, ya sea compartiendo puntos de vista o discutiendo posturas con los miembros de la clase, añadiendo una cuantas frases que describan sus cuatro ideas sobrevenidas. Estas últimas reflexiones pueden demostrar un reforzamiento de los puntos de vista iniciales del alumno o un cambio de opiniones basado en el reconocimiento de la fuerza de los argumentos o experiencias introducidos por la discusión en clase. Todas estas categorías de escritura de diarios proporcionan oportunidades para el intercambio de reacciones de los alumnos frente a la literatura, ampliando así sus experiencias de aprendizaje y aumentando sus bases de conocimiento. Resultan representativas del verdadero conocimiento frente a los tipos superficiales de aprendizaje, puesto que comprometen al alumno a distancia en actividades de pensamiento crítico, en el aprendizaje basado en problemas y, dependiendo de los temas a discutir, estimulan la motivación del alumno en el aprendizaje real del contenido del curso (Boiling, 1994).

\section{EVALUACIÓN DEL APRENDIZAJE DEL ALUMNO}

La cuestión restante se refiere a cómo puede determinar el profesor el aumento y desarrollo del conocimiento del alumno del contenido del curso asignado utilizando la escritura de diarios como tipo principal o, al menos, como un tipo de estrategia educativa. En el proceso descrito de puesta en práctica de la escritura de diarios, los alumnos deben observar una serie de normas y procedimientos para llevar a cabo adecuadamente este método de enseñanza. Fundamentalmente, los alumnos deben seguir los calendarios de tareas establecidos, respetar la longitud y formato de la entrada del diario, completar las tareas con puntualidad, dedicarse de forma adecuada a la interacción con su compañero o 
con un pequeño grupo de miembros de la clase, utilizar el comentario del diario a debatir como base del discurso de la clase durante las sesiones de videoconferencia interactiva, y entregar los diarios terminados al profesor con la petición de que los revise, les dé respuesta y los califique.

La mayor parte de estos procedimientos pueden ser observados por el profesor mediante el contacto periódico con los miembros de la clase a través del correo electrónico o la participación en los grupos de sala de charla como visitante "infrecuente". Además, las cuestiones planteadas por el profesor durante las sesiones de clase a distancia o los análisis y críticas ofrecidos por los integrantes de la clase servirán para proporcionar una evaluación informal del grado en que los alumnos están comprendiendo y aplicando el contenido de la materia que se les ha asignado. No obstante, al utilizar cualquiera de los formatos de escritura de diarios (compañero, etc.), el profesor solicitará copias impresas o remitidas por correo electrónico del comentario diario del alumno, para leerlo y darle respuesta. Aunque este procedimiento se recomienda como resumen final para la evaluación del alumno, la evaluación periódica para comprobar las entradas del diario es importante tanto para el profesor como para el alumno (Peruniak, 1983). La respuesta del profesor proporciona a éste la oportunidad de ofrecer una contestación escrita y permite a los alumnos evaluar la forma en que el profesor percibe su comprensión de la materia. Y viceversa, leer y responder a los diarios del alumno permite al profesor hacerse una idea de la medida en que dicho alumno ha interiorizado la información, es (o ha sido) capaz de aplicarla en el discurso y ha demostrado algún avance y desarrollo desde la anotación inicial del diario a las finales. Este procedimiento ayuda a evaluar si los alumnos están alcanzando con éxito las metas del programa de estudios de postgraduado, desde el momento en que los diarios revelan el pensamiento y creencias del alumno basados en su reacción al contenido de las materias en combinación con sus propias experiencias personales.

La cuestión final se refiere a la forma de calificar el comentario del diario. Los profesores pueden elaborar sus propias normas, que se dan a conocer a los alumnos al inicio del curso; este procedimiento puede funcionar con algunos materiales del curso, pero no con todos. Los profesores pueden elaborar categorías que analicen el comentario del alumno, su cambio de actitud y la evolución de la forma en que éste selecciona y discute los temas. Teniendo en cuenta que la opinión y la reflexión juegan un papel importante en el comentario del diario escrito, el enfoque, de carácter fundamentalmente subjetivo, está compuesto por criterios que determinan qué grado de contenido demuestra haber asimilado el alumno, qué desarrollo en la comprensión de la materia, y en qué medida se ha dedicado al pensamiento crítico, a la investigación y a la conexión de los nuevos conocimientos con los que ya poseía. Como se ha observado en numerosos cursos de enseñanza tanto tradicional como a distancia, parece que el interés del alumno tiende a ser bastante alto durante las actividades compartidas y de escri- 
tura de diarios, y por ello la mayor parte del trabajo de los alumnos está probablemente correlacionado en gran medida con estos criterios.

La descripción de la puesta en práctica de esta técnica de enseñanza en el aprendizaje a distancia debe proporcionar tanto a los profesores como a los alumnos un método viable para interiorizar el aprendizaje del contenido del curso. Como el centro de atención de la mayor parte de la literatura educativa versa sobre los descubrimientos de los investigadores de campo y sobre los informes de prácticas que han tenido éxito, la mayor parte del trabajo del curso puede utilizar con facilidad y éxito algún formato de escritura de diarios en un entorno de videoconferencia de doble enlace. Algunas partes de esta estrategia educativa pueden utilizarse también con audioconferencia combinada con aprendizaje por ordenador, pero esto no puede proporcionar todos los beneficios disponibles. Como abunda la literatura en la mayor parte de las disciplinas, especialmente en el campo de la enseñanza, la escritura de diarios puede convertirse en una técnica óptima para los alumnos de escuelas de postgraduados que lleven a cabo trabajos de un curso de educación a distancia mediante videoconferencia interactiva de doble enlace.

\section{REFERENCIAS BILIOGRÁFICAS}

Bolling, A. (1994). Using group journals to improve writing and comprehension. Journal on Excellence in College Teaching, 5 (1), 47-55.

Brundage, D. Keane, R., \& Mackneson, R. (1993). Application of learning theory to the instruction of adults. En: Thelma Barer-Stein and James A. Draper (Eds.), The craft of teaching adults (pp. 131-144). Toronto, Ontario: Culture Concepts.

Cropley, A. J. \& Kahl, T. N. (1983). Distance education and distance learning: Some psychological considerations. Distance education 4 (1), 27-39.

Dille, B. \& Mezack, M. (1991). Identifying predictors of high risk among community college telecourse students. The American Journal of Distance Education, 5 (1), 24-35.

GARSIDE, C. (1994, November). Building bridges to critical thinking: Utilizing student journal in the college classroom. Informe presentado en la Annual Meeting of the Speech Communication Association, New Orleans, LA.

GotTschalk, T.H. (1996, November). Guide \#3: Instructional Development for Distance Education. University of Idaho: Engineering Outreach. http://www.uidaho.edu/evo/dist3.html

Gunawardena, C. \& Boverie, P. (1995, July). Analysis of learner characteristics and group function in thecnology mediated distance learning. En: A. Tait (Ed.) Putting the student first: Learner-centered approaches in open and distance learning. Sixth Cambridge International Conference in Open and Distance Learning, (pp.65-78). UK: Open University.

Heinze, T. (1983). The social and psychological milieu of distance students. Distance Education, 4 (1), 53-63. 
HUMPHREY, C.F. (1974, April). A study of adults' preferences for control of molar learning activities. Informe presentado en la Adult Education Research Conference. Chicago III (ERIC Document 094103).

Knapper, C. (1988). Lifelong learning and distance education. American Journal of Distance Education, 2 (1), 63-72.

Johnson, A. (1996, February). Curriculum content and affective goals through journal writing in graduate level education courses. Informe presentado en la Eastern Educational Research Association Conference, Hiltonhead, S.C.

Lowman, J. (1995). Mastering Techniques of teaching (Second edition). San Francisco: JosseyBass Publishers.

LudLow, B. (1994, March). A comparison of traditional and distance education models. En: Diane Montgomery (Ed.) Rural partnerships. Working together (pp. 114-121). Proceedings of the Annual National Conference of the American Council on Rural Special Education (ACRES) Austin, Texas. RC 019557.

MolLER, L. (1991). Planning programs for distant learners. Using the Assure model. Tech Trends, 36 (1), 55-57.

Owen, T. (1989). Computer-mediated writing: The writer in electronic residence. En: R. Mason \& A. Kaye (Eds.) Mindweave: Communication, computers and distance education, pp. 321. Oxford: Pergamon Press.

PERRIN, D.G. (1997, March). New knowledge society and higher education. Education at a Distance, 11 (3), 12-20.

REED, J. \& Woodruff, M. (1995). Using compressed video for distance learning. En: The Distance Educator Newsletter, CA: San Diego State University. http://www.kn.pacbell.com/ wired/vidconf/Using.html

Rezabek, L.L. (1997, Under Review). Distance cubed: The psychosocial dimension of distance education.

Seshachari, N.C. (Winter, 1994). Instructor-mediated journals: Raising critical thinking and discourse levels. College Teaching 42 (1).

Threlkeld, R. \& Brzoska, K. (1994). Research in distance education. En: Barry Willis (Ed.) Distance education: Strategies and tools, pp. 41-62. Englewood Cliffs, NJ: Educational Technology Publications.

Von Holzen, R. (1995). Electronic student journals. A means to enhance classroom communications. (ERIC Document Reproduction Service No. ED 392 431).

Willis, B. (1993). Distance education: a practical guide. Englewood Cliffs, NJ: Educational Technology Publications. 


\section{PERFIL ACADÉMICO-PROFESIONAL DEL AUTOR}

Addie M. Johnson, es Assistant Professor de Educación en la Pennsylvania State University, Great Valley Graduated School de Estados Unidos. Durante el curso 1997-98 ha sido Urban Fellow en el Mid-Atlantic Regional Educational Research Laboratory. Su línea de investigación se interesa por las Técnicas de Enseñanza a Distancia, usos de la Multimedia en las escuelas, desarrollo profesional y estándares académicos en el aula. Durante el año académico 1997-98 ha participado a nivel nacional, estatal y regional en la American Educational Research Conferences.

Addie M. Johnson (Ed. D.)

1520 Church Road

Wyncote, PA 19095

USA

Teléf.: 1-215-884-2132 (plus US code)

Fax:1-610-648-3377.

Correo Electrónico: amj8@psu.edu 